\title{
Research Progress of Antitumor Activity by Baicalin and Baicalein
}

\author{
Wang $\mathrm{Yu}^{1}$, Li Ling ${ }^{1}$ \\ ${ }^{1}$ School of Basic Medical Sciences, Qingdao University, 266071, China

\begin{abstract}
Baicalin and its aglycon, baicalein, are flavonoids present from Scutellaria baicalensis Georgi. Baicalin generally inhibits tumor cells by inducing apoptosis and arresting growth, while baicalein suppresses by regulating autophagy. Certain signaling pathways and regulating factors are involved among the induced-apoptosis and autophagy progress. In suppressing growth, baicalin and baicaein inhibit angiogenesis or induce cell cycle arrest at $\mathrm{S}$ phase against proliferation, migration and differentiation. The present agents against tumor still have related side effects. Therefore, the natural and nontoxic flavonoids are promising in present even future tumor treatment.
\end{abstract}

Keywords: Antitumor, Baicalin, Baicalein

\begin{abstract}
1 Introduction
For the past few years, the anticancer activity of baicalin and baicalein has been increasingly paid much attention to. On one hand, cancer has been a well-known serious disease. Cancer is defined as a disease caused by abnormal cell growth that may invade different organs of the body. According to statistics, 14.1 million people suffer from cancer every year in the world.[1, 2] For example, primary hepatic cancer or liver cancer is the second leading cause of cancer-related death; colorectal cancer is the second most fatal type of tumor and affects over 1 million individuals annually worldwide[3, 4].
\end{abstract}

On the other hand, baicalin is one of few natural and nontoxic agents against cancer. Astragalus membranaceus is a traditional Chinese herbal medicine with the effects of clearing away heat and dampness, purging fire and detoxification. At present, more than 40 kinds of flavonoids have been found in Astragalus, among which Baicalein, Wogonin and Baicalin (Baicalin) and Wogonoside are high in Astragalus and have obvious pharmacological effects. Previous phytochemical studies showed that the major flavonoids in Scutellaria baicalensis are baicalin (baicalein-7-O-gluc-uronide), baicalein (5,6,7-trihydoxyflavone)[5], wogonoside and wogonin, the glycoside (baicalin)[6] can be transformed into its aglycon (baicalein) [7, 8]. Both of them possess significant pharmacological activities[9], and have great potency with less toxicity in inhibiting hypertension, inflammation, allergies and hepatitis, as well as cancer[10] including lung, colorectal, prostate and breast carcinomas[10-14].
2 The role of baicalin and baicalein in different cancer mechanism

2.1 Potent inhibitors of angiogenesis

MMP-2 and MMP-9 are key molecules in the degradation of the extracellular matrix, which affect the metastasis of cancer cells by inducing angiogenesis. In addition, the protein kinase B (AKT) pathway is crucial in the invasion of cancer cells through the regulation MMPs-2 and $-9[3,15]$.

Liu, J. J. et[16] assessed baicalin and baicalein as anti-angiogenic agents in vivo employing chicken chorioallantoic membrane (CAM) assay and in vitro human umbilical vein endothelial cells (HUVECs) culture. They also explored the mechanism of antiangiogenesis through following progress: cell proliferation assay, zymogram analysis for MMP-2, cell migration assay and endothelial tube formation assay on matrigel .The results showed that the antiangiogenic mechanisms of the two flavonoids involve that cell-associated MMP-2 activity are reduced, migration and proliferation are inhibited, and in vitro vascular endothelial cells form capillary[16, 17].

Rui, X. et al[3] and Choi, E. O. et al[15] reported baicalein appears to suppress colorectal cancer cell migration and invasion by decreasing the expression of MMP-2 and MMP-9 via inhibition of the AKT signaling pathway.

\section{2 cell cycle arrest}

According to LEE, H. Z. et al[18] and Yang $\mathrm{Yu}$ et al[2] research, we can find that both flavonoids induce cell cycle arrest of $\mathrm{S}$ phase to suppress cancer cells such as hepatic cancer, human lung cancer cells proliferation and development.

This article is published under the terms of the Creative Commons Attribution License 4.0 Author(s) retain the copyright of this article. Publication rights with Alkhaer Publications. Published at: http://www.ijsciences.com/pub/issue/2019-06/

DOI: 10.18483/ijSci.2099; Online ISSN: 2305-3925; Print ISSN: 2410-4477 
The $\mathrm{S}$ phase is associated with DNA synthesis and the mitotic preparation period, which plays a crucial role in cell cycle progression. Cyclin A and CDK2 form a complex as the mitosis-promoting factor, which is thought to be the key controller of the progress from $\mathrm{S}$ phase to mitosis[2].

Yang $\mathrm{Yu}$ et al[2] clarified that baicalin-treatment downregulates the complex expresssion to $\mathrm{S}$ phase arrest, so that cancer cells are inhibited to grow. This study demonstrated through colony formation assay, flow cytometry analysis of cell cycle, hoechst 33342 staining and Western blot analysis.

\subsection{Apoptosis}

2.3.1 Cooperative effects of baicalein and baicalin The extrinsic and intrinsic apoptotic pathways converge to caspase- 3 . And in the intrinsic pathway, the upstream of caspase-3 is caspase-9. Apoptosis involves certain death and survival gene such as bcl-2 and bax[10, 19-21].

Zhou, Q. M. et al [19] reported the combination of baicalin and baicalein enhances apoptosis in human breast cancer cells. Caspase- 3 and caspase-9 are activated, bcl-2 is up-regulated[10] and bax is downregulated by the combination via the ERK/p38 MAPK pathway.

\subsubsection{Single effect of baicalin}

Baicalin-treatment down-regulates the expression levels of CDK2, Cyclin A, and Cyclin D1[2]. Moreover, baicalin induces apoptotic cell death, which is accompanied with up-regulation of Bax, down-regulation of $\mathrm{Bcl}-2$ [22], and cleavages of Caspase-9, Caspase-3, and PARP[2].

Chen,W.-C. et al[22] demonstrated that baicalintreatment of human colorectal carcinoma cells enhances the activity of caspases in the intrinsic and extrinsic apoptosis pathways, accompanying the activation of caspase- $3,-8$, and -9 .

The expression of the pro-apoptotic protein Bax is up-regulated by baicalin treatment in both Hep G2 and SMMC-7721 cell lines, while the expression of Bcl-2 which belongs to anti-apoptotic proteins is down-regulated. What's more, the protein levels of cleaved-Caspase-3, cleaved-Caspase- 9 and cleavedPARP1 are up-regulated[2] via PI3K/Akt signal pathways[23].

\subsection{Autophagic cell death}

Autophagy is a normal catabolic process to degrade damaged organelles and dysfunctional proteins and maintain cellular homeostasis[10]. ULK1 is a serine/threonine kinase, which acts downstream of the autophagy inhibitor mTOR and forms a large complex with Atg13 and FIP200 to initiate autophagy[24].
Aryal, P. et al[10] put a new mechanism that baicalein suppresses the growth of human cancer cells inducing autophagic cell death in human prostate and breast cancer cells. The progress involves AMP-activated protein kinase (AMPK) ULK1 activation through modulating the expression level of anti-autophagic molecules such as mTOR and Raptor. And it was the first time to investigate whether the antitumor properties of baicalin is mediating cancer cells autophagy.

The plasticity of tumor-associated macrophages (TAMs) has an influential role that favours tumour regression on hepatocellular carcinoma (HCC)[25].Tan, H. Y. et al[25] talked about the role and mechanism of TAM repolarisation caused by natural compound baicalin in suppression of HCC. They found that baicalin induced repolarization of TAM to M1-like phenotype without specific toxicity, and promoted pro-inflammatory cytokines production; baicalin-treated TAMs co-culturing with HCC suppressed proliferation and motility in $\mathrm{HCC}$; the effect was associated with elevated autophagy, and transcriptional activation of RelB/p52 pathway[25].

\subsection{Inhibiting the proliferation and invasion}

The degradation of the ECM of blood or lymph vessels is one of the key steps in metastasis, which involves MMP-2 and $-9[3,26,27]$.

Rui, X. et al [3] were the first to investigate the antimetastatic effect of baicalein on CRC cells, and they investigated the hypothesis that treatment with baicalein arrest the motility, proliferation and invasion of human CRC cell lines by reducing the expression of MMP-2 and MMP-9 via regulation of the protein kinase B (AKT) signaling pathway. Thus, baicalein is a potential new therapeutic agent for patients with CRC.

Choi, E. O. et al.[15] found that baicalein significantly inhibits invasiveness and the cell motility of B16F10 mouse melanoma Cells within the noncytotoxic concentration range, which is associated with its tightening of tight junction (TJ). The facts were demonstrated by down-regulation of the claudin family of proteins and increase in transepithelial electrical resistance. Moreover, baicalein also markedly decrease the expression levels of lipopolysaccharide-induced phosphorylated Akt.

\section{Pretreatment of baicalin and wogonoside}

$\mathrm{Yu}, \mathrm{C}$. et al[7] used glycosidase to catalyze baicalin and wogonoside to enhance the anticancer activities. The HPLC data showed that there was a marked transformation from the two glycosides to their aglycons $\left(20 \mathrm{U} / \mathrm{g}\right.$ of cellulase, $50^{\circ} \mathrm{C}, \mathrm{pH} 4.8$ and treatment for $8 \mathrm{~h}$ ). 
They evaluated the anticancer activity by a series of $\mathrm{S}$. baicalensis extracts in which variable lengths of glycosidase treatment time were used. And the results were the antiproliferation effects were stronger with the higher the aglycon content. The conclusion was clarified after experiments on human colorectal and breast cancer cells that glycosidase offers a promising approach to enhance the anticancer activity of baicalin and wogonoside.

\section{Novel forms of baicalin or baicalein 4.1 BA-j}

8-Hydroxypiperidinemethyl-baicalein (BA-j) is a new selective CDK1 inhibitor with broad spectrum antitumor activity (IC50 $12.3 \mu \mathrm{M}$ ) and 2 tumor xenografts[7]. BA-j can promote both the extrinsic death receptor pathway and the intrinsic apoptosis pathway, which is different from cytotoxic antitumor drugs, and therefore kill cancer cells rather than normal cells.

\subsection{NPs for combination of PTX and BCL prodrugs}

Wang, W. et al[28] developed a dual-ligand, multifunctional, self-assembled, modified nanoparticles (NPs) for the combination delivery of paclitaxel (PTX) and baicalein (BCL) prodrugs. The effect of prepared PTX-BCL NP drug-delivery system was proven by its delivering of BCL and PTX prodrugs and targeting of drug-resistant human lung cancer cells.

\section{Conclusion}

In general, baicalein and baicalin have multiple anticancer effects. They regulate almost all types of cancer, including cell proliferation, cancer signaling pathways, transcription factors, and tumor angiogenesis. In the process of cell proliferation, it targets the overexpression of CDK2 by inhibiting the cell cycle, thereby achieving the purpose of inhibiting CDK2 activity. In addition, it promotes p53 function in many wild and mutant p53 tumor cell lines. Baicalin and baicalein regulate a variety of tumor proteins involved in tumor signaling, for example by inhibiting the growth of many tumor cells by targeting the $\mathrm{P} 13 \mathrm{~K}$, AKT, Ras and $\beta$-catenin pathways. Baicalin and baicalein also inhibit several key molecules of tumor angiogenesis and metastasis, including VEGF, MMP and PTPN. In conclusion, baicalien and bacalin are excellent diet for the treatment and prevention of different types of cancer.

\footnotetext{
References

1. Wang, C. Z., Zhang, C. F., Chen, L., Anderson, S., Lu, F. \& Yuan, C. S. (2015) Colon cancer chemopreventive effects of baicalein, an active enteric microbiome metabolite from baicalin, International journal of oncology. 47, 174958.doi:10.3892/ijo.2015.3173

2. Yang Yu, M. P., Ling Li (2015) Baicalin induces apoptosis in
}

hepatic cancer cells in vitro and suppresses tumor growth in vivo, Int J Clin Exp Med. 8, 10

3. Rui, X., Yan, X. I. \& Zhang, K. (2016) Baicalein inhibits the migration and invasion of colorectal cancer cells via suppression of the AKT signaling pathway, Oncology letters. 11, 685-688.doi:10.3892/ol.2015.3935

4. Morere, L., Andouard, D., Labrousse, F., Saade, F., Calliste, C. A., Cotin, S., Aubard, Y., Rawlinson, W. D., Esclaire, F., Hantz, S., Ploy, M. C. \& Alain, S. (2015) Ex vivo model of congenital cytomegalovirus infection and new combination therapies, $\quad$ Placenta. 36, 41 7.doi:10.1016/j.placenta.2014.11.003

5. Sahu, B. D., Mahesh Kumar, J. \& Sistla, R. (2015) Baicalein, a Bioflavonoid, Prevents Cisplatin-Induced Acute Kidney Injury by Up-Regulating Antioxidant Defenses and DownRegulating the MAPKs and NF-kappaB Pathways, PLoS One. 10, e0134139.doi:10.1371/journal.pone.0134139

6. Wang, C. Z., Calway, T. D., Wen, X. D., Smith, J., Yu, C. Wang, Y., Mehendale, S. R. \& Yuan, C. S. (2013) Hydrophobic flavonoids from Scutellaria baicalensis induce colorectal cancer cell apoptosis through a mitochondrialmediated pathway, International journal of oncology. 42, 1018-26.doi:10.3892/ijo.2013.1777

7. Yu, C., Zhang, Z., Zhang, H., Zhen, Z., Calway, T., Wang, Y., Yuan, C. S. \& Wang, C. Z. (2013) Pretreatment of baicalin and wogonoside with glycoside hydrolase: a promising approach to enhance anticancer potential, Oncology reports. 30, 2411-8.doi:10.3892/or.2013.2726

8. Shin, H. S., Bae, M. J., Choi, D. W. \& Shon, D. H. (2014) Skullcap (Scutellaria baicalensis) extract and its active compound, wogonin, inhibit ovalbumin-induced Th2mediated response, Molecules. 19, 253645.doi:10.3390/molecules19022536

9. Yong Peng, Z.-z. F., Cong-Shan Guo (2015) Effects and Mechanism of Baicalin on Apoptosis of Cervical Cancer HeLa Cells In-vitro, Iranian Journal of Pharmaceutical Research. 14, 12

10. Aryal, P., Kim, K., Park, P. H., Ham, S., Cho, J. \& Song, K. (2014) Baicalein induces autophagic cell death through AMPK/ULK1 activation and downregulation of mTORC1 complex components in human cancer cells, The FEBS journal. 281, 4644-58.doi:10.1111/febs.12969

11. Chen, J., Li, Z., Chen, A. Y., Ye, X., Luo, H., Rankin, G. O. $\&$ Chen, Y. C. (2013) Inhibitory effect of baicalin and baicalein on ovarian cancer cells, Molecular Sciences. 14, 6012-25.doi:10.3390/ijms14036012

12. Wang, T., Shi, G., Shao, J., Wu, D., Yan, Y., Zhang, M., Cui, Y. \& Wang, C. (2015) In vitro antifungal activity of baicalin against Candida albicans biofilms via apoptotic induction, $\begin{array}{lll}\text { Microbial pathogenesis. } & 87, & \end{array}$ 9.doi:10.1016/j.micpath.2015.07.006

13. Liao, H. L. \& Hu, M. K. (2004) Synthesis and anticancer activities of 5,6,7-trimethylbaicalein derivatives,

14. Zhang, D. Y., Wu J Fau - Ye, F., Ye F Fau - Xue, L., Xue L Fau - Jiang, S., Jiang S Fau - Yi, J., Yi J Fau - Zhang, W., Zhang W Fau - Wei, H., Wei H Fau - Sung, M., Sung M Fau - Wang, W., Wang W Fau - Li, X. \& Li, X. (2003) Inhibition of cancer cell proliferation and prostaglandin E2 synthesis by Scutellaria baicalensis,

15. Choi, E. O., Cho, E. J., Jeong, J. W., Park, C., Hong, S. H., Hwang, H. J., Moon, S. K., Son, C. G., Kim, W. J. \& Choi, Y. H. (2016) Baicalein Inhibits the Migration and Invasion of B16F10 Mouse Melanoma Cells through Inactivation of the PI3K/Akt Signaling Pathway, Biomolecules \& therapeutics.doi:10.4062/biomolther.2016.094

16. Liu, J. J., Huang, T. S., Cheng, W. F. \& Lu, F. J. (2003) Baicalein and baicalin are potent inhibitors of angiogenesis: Inhibition of endothelial cell proliferation, migration and differentiation, International journal of cancer. 106, 559. 65.doi:10.1002/ijc.11267

17. Wu, J. Y., Tsai, K. W., Li, Y. Z., Chang, Y. S., Lai, Y. C. Laio, Y. H., Wu, J. D. \& Liu, Y. W. (2013) Anti-BladderTumor Effect of Baicalein from Scutellaria baicalensis 
Georgi and Its Application In Vivo, Evidence-based complementary and alternative medicine : eCAM. 2013, 579751.doi:10.1155/2013/579751

18. LEE, H. Z., LEUNG, H. W. C., LAI, M. Y. \& WU, C. H. (2005) Baicalein Induced Cell Cycle Arrest and Apoptosis in Human Lung Squamous Carcinoma CH27 Cells, Anticancer Research. 25, 959-964

19. Zhou, Q. M., Wang, S., Zhang, H., Lu, Y. Y., Wang, X. F., Motoo, Y. \& Su, S. B. (2009) The combination of baicalin and baicalein enhances apoptosis via the ERK/p38 MAPK pathway in human breast cancer cells, Acta pharmacologica Sinica. 30, 1648-58.doi:10.1038/aps.2009.166

20. Chen, Y. J., Wu, C. S., Shieh, J. J., Wu, J. H., Chen, H. Y., Chung, T. W., Chen, Y. K. \& Lin, C. C. (2013) Baicalein Triggers Mitochondria-Mediated Apoptosis and Enhances the Antileukemic Effect of Vincristine in Childhood Acute Lymphoblastic Leukemia CCRF-CEM Cells, Evidence-based complementary and alternative medicine : eCAM. 2013, 124747.doi:10.1155/2013/124747

21. Lee, J. H., Li Yc Fau - Ip, S.-W., Ip Sw Fau - Hsu, S.-C., Hsu Sc Fau - Chang, N.-W., Chang Nw Fau - Tang, N.-Y., Tang Ny Fau - Yu, C.-S., Yu Cs Fau - Chou, S.-T., Chou St Fau Lin, S.-S., Lin Ss Fau - Lino, C.-C., Lino Cc Fau - Yang, J.S., Yang Js Fau - Chung, J.-G. \& Chung, J. G. The role of $\mathrm{Ca} 2+$ in baicalein-induced apoptosis in human breast MDAMB-231 cancer cells through mitochondria- and caspase-3dependent pathway,

22. Chen, W.-C., Kuo, T.-H., Tzeng, Y.-S. \& Tsai, Y.-C. (2012) Baicalin Induces Apoptosis in SW620 Human Colorectal Carcinoma Cells in Vitro and Suppresses Tumor Growth in Vivo, Molecules. 17, 38443857.doi:10.3390/molecules17043844

23. Yi Huang, J. H., , Jing Zheng (2012) Down-regulation of the PI3K/Akt signaling pathway and induction of apoptosis in
CA46 Burkitt lymphoma cells by baicalin, Journal of Experimental \& Clinical Cancer Research. 31, 9

24. Hosokawa, N., Hara, T., Kaizuka, T., Kishi, C., Takamura, A., Miura, Y., Iemura, S., Natsume, T., Takehana, K. Yamada, N., Guan, J. L., Oshiro, N. \& Mizushima, N. (2009) Nutrient-dependent mTORC1 association with the ULK1Atg13-FIP200 complex required for autophagy, Molecular biology of the cell. 20, 1981-91.doi:10.1091/mbc.E08-121248

25. Tan, H. Y., Wang, N., Man, K., Tsao, S. W., Che, C. M. \& Feng, Y. (2015) Autophagy-induced RelB/p52 activation mediates tumour-associated macrophage repolarisation and suppression of hepatocellular carcinoma by natural compound baicalin, Cell death \& disease. 6 , e1942.doi:10.1038/cddis.2015.271

26. Peng, X. D., Dai, L. L., Huang, C. Q., He, C. M. \& Chen, L. J. (2009) Correlation between anti-fibrotic effect of baicalin and serum cytokines in rat hepatic fibrosis, World journal of gastroenterology. 15, 4720-5.doi:10.3748/wjg.15.4720

27. Ma, Z., Otsuyama K Fau - Liu, S., Liu S Fau - Abroun, S., Abroun S Fau - Ishikawa, H., Ishikawa H Fau - Tsuyama, N., Tsuyama N Fau - Obata, M., Obata M Fau - Li, F.-J., Li Fj Fau - Zheng, X., Zheng X Fau - Maki, Y., Maki Y Fau Miyamoto, K., Miyamoto K Fau - Kawano, M. M. \& Kawano, M. M. Baicalein, a component of Scutellaria radix from Huang-Lian-Jie-Du-Tang (HLJDT), leads to suppression of proliferation and induction of apoptosis in human myeloma cells,

28. Wang, W., Xi, M., Duan, X., Wang, Y. \& Kong, F. (2015) Delivery of baicalein and paclitaxel using self-assembled nanoparticles: synergistic antitumor effect in vitro and in vivo, International journal of nanomedicine. 10, 3737 50.doi:10.2147/IJN.S80297 